\title{
Triazole Acetyl Gold(III) Catalyzed Meyer-Schuster Rearrangement of Propargyl Alcohols
}

\author{
Yongchun Yang, ${ }^{a}$ Yanan Shen, ${ }^{a}$ Xiaoli Wang, ${ }^{a}$ Yao Zhang, ${ }^{a}$ Dawei Wang* ${ }^{a}$ and Xiaodong Shi* ${ }^{b}$ \\ ${ }^{a}$ The Key Laboratory of Food Colloids and Biotechnology, Ministry of Education, School of Chemical and Material Engineering, Jiangnan \\ University, Wuxi 214122, Jiangsu Province, China. \\ ${ }^{b} 4202$ E Fowler Ave, Department of chemistry, University of South Florida, Tampa, Florida, USA. \\ Email: wangdw@jiangnan.edu.cn, xmshi@usf.edu, Tel./fax: +86 51085917763
}

\section{ARTICLE INFO \\ ABSTRACT}

Article history:

Received

Received in revised form

Accepted

Available online

Keywords:

triazole,

gold,

Meyer-Schuster rearrangement,

propargyl alcohols,

haloenones

\begin{abstract}
A new type triazole acetyl gold(III) was prepared and found to be an effective catalyst in Meyer-Schuster rearrangement of propargyl alcohols. The reactions proceeded well under much milder conditions to afford enones bearing a wide range of functional groups, thereby opening a new avenue for gold(III) catalysis. In addition, TriaAuCl${ }_{2}$ catalyst was also effective on promotion of ahaloenones synthesis.
\end{abstract}

\section{Introduction}

Gold catalysis has been widely applied in organic transformations, and methods for their efficient, selective functionalization to construct more complex molecules during the past several decades. ${ }^{1,2}$ Gold(I) catalysts are usually two coordinated with $180^{\circ}$ linear geometry, while gold(III) catalysts have a planar coordination geometry, and hence the spatial environment around the gold center can be more easily fine-tuned through ligand design studies. However, gold(I) catalysis has achieved much more development and concern than gold(III) catalysis, which should be attributed to the heat stability of gold catalyst. ${ }^{3}$ The typical gold(III) catalyst is the $\mathrm{PicAuCl}_{2}$ and their derivatives, which enriched and evidenced the development of gold(III) catalysis (Scheme 1A). After some pioneering works on $\mathrm{PiCAuCl}_{2},{ }^{4}$ Hashmi reported $\mathrm{PicAuCl}_{2}$ catalyzed phenol synthesis, which revealed that gold(III) precatalysts represents a typical step in catalysttuning by ligand design, while they found $\mathrm{Au}(\mathrm{I})$ showed low selectivity. ${ }^{5}$ Toste et al described a $\mathrm{PicAuCl}_{2}$ catalyzed synthesis of azepines via intermolecular [4 +3]-annulation reaction. ${ }^{6}$ In 2013, Waser et al developed the selective synthesis of 2- and 3-alkynylated furans based on a domino cyclization/alkynylation process with $\mathrm{PicAuCl}_{2}$ as catalyst. ${ }^{7}$ Chan group showed that $\mathrm{PicAuCl}_{2}$ catalyzed selective 1,3acyloxy migration/5-exo-dig cyclization/1,5-acyl migration to afford cis-cyclopenten-2-yl $\delta$-diketones. ${ }^{8}$
2015 Elsevier Ltd. All rights reserved.
Scheme 1. $\mathrm{PicAuCl}_{2}$ and $\mathrm{TriaAuCl}$.

$1 \mathrm{~A}$<smiles>O=C1ON(Cl)C2CCCCC12</smiles>

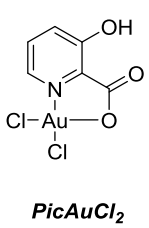<smiles></smiles>
$1 \mathrm{~B}$<smiles>O=C(Cn1nnc2ccccc21)N1CC(Cl)(Cl)C1(Cl)Cl</smiles>

Our research in developing new triazole ligands to balance the stability and reactivity of gold catalysts has led to the recent discovery of triazole gold(I) complexes (TA-Au), ${ }^{9,10}$ which was achieved several applications on hydroamination, Hashmi phenol synthesis and 3,3-rearrangements with TA$\mathrm{Au}(\mathrm{I})$ as a catalyst. We have a concern about triazole ligands: could triazole adjust the stability and reactivity of gold catalysts gold(III)? Herein, we synthesized the TriaAu(III)Cl $\mathrm{Cl}_{2}$ complex, which showed excellent catalytic activity in MeyerSchuster rearrangement of propargyl alcohols for enone synthesis under mild conditions, which avoid the preparation of corresponding acetate derivatives and have large substrates exploration (Scheme 1B).

Scheme 2. The synthesis of TriaAu(III) $\mathrm{Cl}_{2}$ complex. 


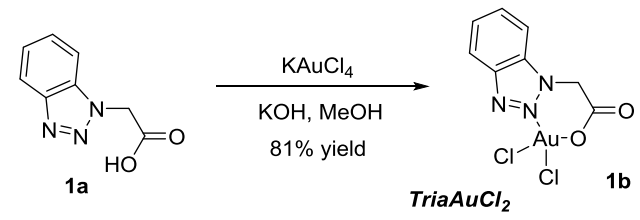

The designed (triazol-1-yl)acetic acid (1a) was synthesized from benzotriazole and methyl 2-bromoacetate via two steps with $86 \%$ yield $^{11}$ following dealt with potassium tetrachloroaurate under base conditions and the obtained TriaAu(III)Cl $\mathrm{Cl}_{2}$ complex (1b) was obtained in $81 \%$ yield. ${ }^{12}$ Next, we explored the catalytic activity of this new catalyst TriaAu(III) $\mathrm{Cl}_{2}$. Compared to the 3,3'-rearrangement of propargyl esters, ${ }^{13}$ Meyer-Schuster rearrangement of propargyl alcohols is quite difficult. Propargyl alcohol (2a) was selected as a model substrate for reaction screening. It is very disappointed that the hydration product was found only with low yield (Table 1, Entry 1). Based on our previous results on $\mathrm{Au}(\mathrm{I}),{ }^{14}$ we firstly examined the effect of solvents on the reactivity using TriaAuCl 2 as the catalyst. As shown in Table 1, the reaction was strongly solvent-dependent. Excellent reactivity was achieved in $\mathrm{MeOH}$. It should be noted that the Meyer-Schuster rearrangement of propargyl alcohols could not take place in the absence of gold catalyst.

Table 1. Screening of reaction conditions ${ }^{a}$

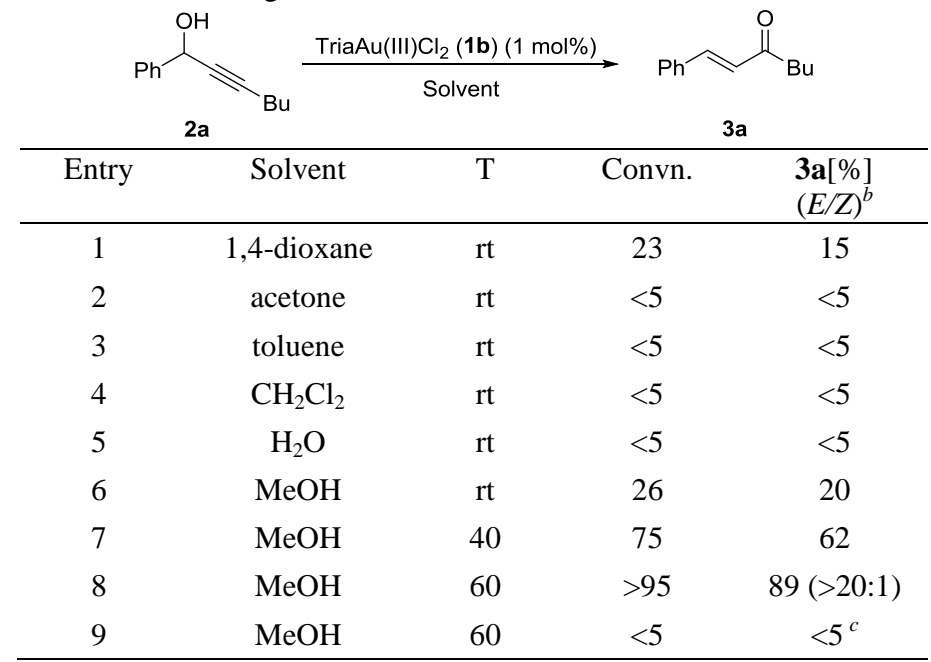

${ }^{a}$ Conditions: 2a $(0.5 \mathrm{mmol})$, TriaAu(III)Cl ${ }_{2}(\mathbf{1 b})(1 \mathrm{~mol} \%)$, wet solvent $\left(10 \% \mathrm{H}_{2} \mathrm{O}\right), 6$ h. ${ }^{b}$ Isolated yields based on $\mathbf{2 a} .{ }^{c} \mathrm{No}[\mathrm{Au}]$ was used.

Having established the optimal conditions, the substrate scope of $\mathrm{TriaAuCl}_{2}$ catalyzed Meyer-Schuster rearrangement of propargyl alcohols was examined. The reactions were carried out in $\mathrm{MeOH}\left(10 \% \mathrm{H}_{2} \mathrm{O}\right)$ at $60{ }^{\circ} \mathrm{C}$. Generally, all the propargyl alcohols were converted completely into the corresponding enones. High yields were achieved regardless of the electronic properties and steric hindrance of substituent groups (Table 2). The propargyl alcohols with $\mathrm{MeO}$ group (3m) gave the highest yield, while a slightly lower yield was obtained when the nitro substituent was in the substrate (31).

Table 2. Substrate expansion of propargyl alcohols ${ }^{a, b}$

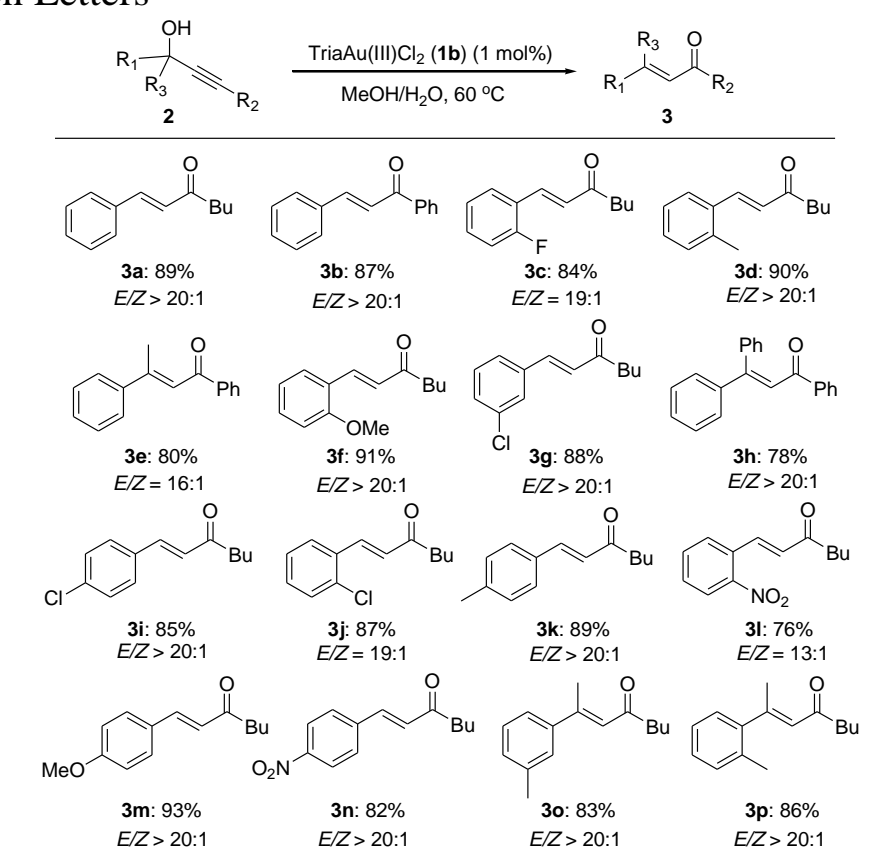

${ }^{a}$ Conditions: $2(0.5 \mathrm{mmol}), \mathrm{TriaAu}(\mathrm{III}) \mathrm{Cl}_{2}(\mathbf{1 b})(1 \mathrm{~mol} \%), \mathrm{MeOH}$ $\left(10 \% \mathrm{H}_{2} \mathrm{O}\right), 60{ }^{\circ} \mathrm{C}, 6$ h. ${ }^{b}$ Isolated yields based on 2 .

In addition, we attempted the experiment with alkylsubstituted propargyl alcohol as a substrate. As illustrated in Scheme 3, the alkyl-substituted enone was achieved in only E isomer $81 \%$ yield.

Scheme 3. Substrate experiment with alkyl-substituted propargyl alcohol.

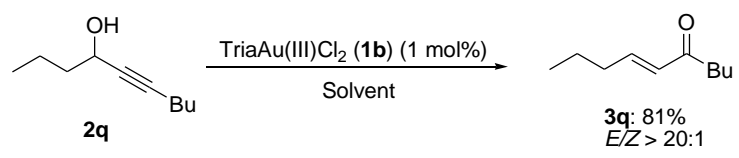

Moreover, TriaAu(III) $\mathrm{Cl}_{2}$ was also an effective catalyst on a-haloenones synthesis, ${ }^{15}$ although a slight lower yield was achieved (Table 3). It was found that the electronic and steric natures of aromatic ring of propargyl esters had no obvious effect on the results. For example, the propargyl esters bearing electron-donating (like $\mathrm{Me}, \mathrm{MeO}$ ) or electron-withdrawing groups at para-, meta-, or ortho-positions were well tolerated, and afforded the corresponding products in moderate yields. Notably, this transformation didn't work for the terminal alkyne since the 1,2-rearramgement was usually preferred for terminal alkyne propargyl acetate.

Table 3. Substrate expansion of propargyl esters ${ }^{a, b}$

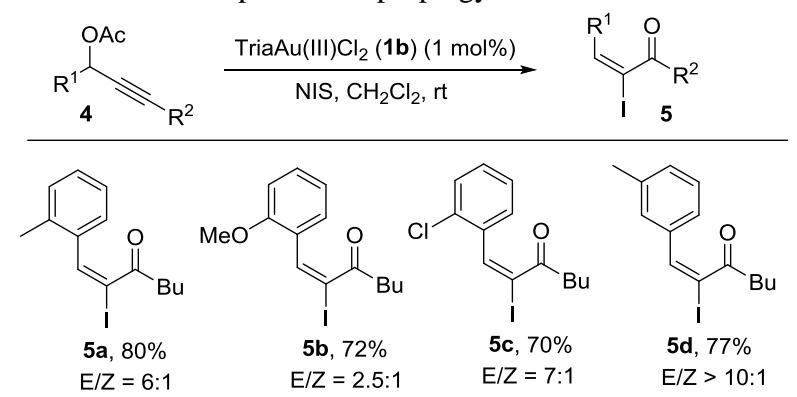

${ }^{a}$ Conditions: 4 (0.5 mmol), NIS (0.75 mmol), TriaAu(III)Cl ${ }_{2}(\mathbf{1 b})(1$ $\mathrm{mol} \%), \mathrm{CH}_{2} \mathrm{Cl}_{2}(3 \mathrm{~mL}), 12 \mathrm{~h}$, rt. ${ }^{b}$ Isolated yields based on 4 . 


\section{Conclusions}

In summary, we developed a new type $\mathrm{TriaAu}(\mathrm{III}) \mathrm{Cl}_{2}$ complex, which showed good catalytic activity in MeyerSchuster rearrangement of propargyl alcohols for enone synthesis under mild conditions, which avoid the preparation of corresponding acetate derivatives and have large substrates exploration. In addition, $\mathrm{TriaAuCl}_{2}$ complex was also effective on promotion of a-haloenones synthesis. Further applications of $\mathrm{TriaAu}(\mathrm{III}) \mathrm{Cl}_{2}$ catalyst to other reactions for the construction of carbon-heteroatom bonds are currently in progress in our laboratory.

\section{Supporting Information}

Supplementary data related to this paper is available free of charge via the Internet.

\section{Acknowledgements}

We gratefully acknowledge financial support of this work by NSF (CHE-1362057), NSFC (21401080, 51503086), NSFC of Jiangsu (BK20130125), CPSF (2014M550262, 2015T80495) and MOE \& SAFEA for the 111 Project (B13025).

\section{References and Notes}

1. For selected recent reviews of homogeneous Au catalysis, see: (a) Fürstner, A.; Davies, P. W. Angew. Chem., Int. Ed. 2007, 46, 3410. (b) Gorin, D.; Toste, F. D. Nature 2007, 446, 395. (c) Corma, A.; Leyva-Pérez, A.; Sabater, M. Chem. Rev. 2011, 111, 1657. (d) Rudolph, M.; Hashmi, A. S. K. Chem. Soc. Rev. 2012, 41, 2448. (e) Liu, L.-P.; Hammond, G. B. Chem. Soc. Rev. 2012, 41, 3129. (f) Garayalde, D.; Nevado, C. ACS Catal. 2012, 2, 1462. (g) Obradors, C.; Echavarren, A. M. Chem. Commun. 2014, 50, 16. (h) Soriano, E.; Fernández, I, Chem. Soc. Rev. 2014, 43, 3041. (i) Zhu, Y.; Sun, L.; Lu, P.; Wang, Y. ACS Catal. 2014, 4, 1911. (j) Jones, A. C.; May, J. A.; Sarpong, R.; Stoltz, B. M. Angew. Chem. Int. Ed. 2014, 53, 2556. k) Dorel, R.; Echavarren, A. M. Chem. Rev. 2015, 115, 9028.

2. Recent examples on gold catalysis: (a) Dorel, R.; Echavarren, A. M. Chem. Rev. 2015, 115, 9028. (b) Winston, M. S.; Wolf, W. J.; Toste, F. D. J. Am. Chem. Soc. 2015, 137, 7921. (c) Veenboer, R. M. P.; Dupuy, S.; Nolan, S. P. ACS Catal. 2015, 5, 1330. (d) Larsen, M. H.; Houk, K. N.; Hashmi, A. S. K. J. Am. Chem. Soc. 2015, 137, 10668. (e) Wang, Y.; Zheng, Z.; Zhang, L. J. Am. Chem. Soc. 2015, 137, 5316. (f) Rao, W.; Susanti, D.; Ayers, B. J.; Chan, P. W. H. J. Am. Chem. Soc. 2015, 137, 6350. (g) Mohamed, R.; Mondal, S.; Gold, B.; Evoniuk, C. J.; Banerjee, T.; Hanson, K.; Alabugin, I. V. J. Am. Chem. Soc. 2015, 137, 6335.

3. Wang, D.; Zhang, Y.; Cai, R.; Shi, X. Beilstein J. Org. Chem. 2011, 7, 1014.

4. (a) Adams, H.-N.; Strähle, J.; Z. Anorg. Allg. Chem. 1982, 485, 65. (b) Adams, H.-N.; Hiller, W.; Strähle, J. Z. Anorg. Allg. Chem. 1982, 485, 81.

5. (a) Hashmi, A. S. K.; Weyrauch, J. P.; Rudolph, M.; Kurpejović, E. Angew. Chem. Int. Ed. 2004, 43, 6545. (b) Hashmi, A. S. K.; Rudolph, M.; Weyrauch, J. P.; Wölfle, M.; Frey, W.; Bats, J. W. Angew. Chem. Int. Ed. 2005, 44, 2798.

6. Shapiro, N. D.; Toste, F. D. J. Am. Chem. Soc. 2008, 130, 9244.
7. Li, Y.; Brand, J. P.; Waser, J. Angew. Chem. Int. Ed. 2013, 52, 6743.

8. Li, D.; Rao, W.; Tay, G. L.; Ayers, B. J.; Chan, P. W. H. J. Org. Chem. 2014, 79, 11301-11315.

9. (a) Duan, H.; Sengupta, S.; Petersen, J. L.; Akhmedov, N. G.; Shi, X. J. Am. Chem. Soc. 2009, 131, 12100. (b) Chen, Y.; Yan, W.; Akhmedov, N.; Shi, X. Org. Lett. 2010, 12, 344. (c) Wang, D.; Ye, X.; Shi, X. Org. Lett. 2010, 12, 2088.

10. For other examples in our group, see: (a) Wang, D.; Cai, R.; Sharma, Jirak, J.; Thummanapelli, S. K.; Akhmedov, N. G.; Zhang, H.; Liu, X.; Petersen, J.; Shi, X. J. Am. Chem. Soc. 2012, 134, 9012.(b) Peng, H.; Xi, Y.; Ronaghi, N.; Dong, B.; Akhmedov, N. G.; Shi, X. J. Am. Chem. Soc. 2014, 136, 13174. (c) Xi, Y.; Su, Y.; Yu, Z.; Dong, B.; McClain, E. J.; Lan, Y.; Shi, X. Angew. Chem., Int. Ed. 2014, 53, 9817. (d) Wang, Q.; Motika, S. E.; Akhmedov, N. G.; Petersen, J. L.; Shi, X. Angew. Chem., Int. Ed. 2014, 53, 5418. (e) Peng, H.; Akhmedov, N. G.; Liang, Y.; Jiao, N.; Shi, X. J. Am. Chem. Soc. 2015, 137, 8912. (f) Cai, R.; Lu, M.; Aguilera, E. Y.; Xi, Y.; Akhmedov, N. G.; Petersen, J. L.; Chen, H.; Shi, X. Angew. Chem., Int. Ed. 2015, 54, 8772. (g) Qin, C.; Su, Y.; Shen, T.; Shi, X.; Jiao, N. Angew. Chem., Int. Ed. 2016, 55, 350.

11. (a) Sengupta, S.; Duan, H.; Lu, W.; Petersen, J. L.; Shi, X. Org. Lett. 2008, 10, 1493. (b) Chen, Y.; Liu, Y.; Petersen, J. L.; Shi, X. Chem. Commun. 2008, 3254. (c) Liu, Y.; Yan, W.; Chen, Y.; Petersen, J. L.; Shi, X. Org. Lett. 2008, 10, 5389. (d) Duan, H.; Yan, W.; Sengupta, S.; Shi, X. Bioorg. Med. Chem. Lett. 2009, 19, 3899. (e) Yan, W.; Wang, Q.; Chen, Y.; Petersen, J. L.; Shi, X. Org. Lett.2010, 12, 3308. (f) Wang, D.; Zhao, K.; Xu, C.; Miao, H.; Ding, Y. ACS Catal. 2014, 4, 3910.

12. TriaAu(III)Cl $\mathrm{Cl}_{2}$ complex (1b) was the fine powder, which was characterized by NMR and elemental analysis but failed to cultivate the crystal, please see the supplementary material for details.

13. For selected examples for 1,3-shifts, see: (a) Y. Chen, M. Chen, Y. Liu, Angew. Chem. Int. Ed. 2012, 51, 6493. (b) J. W. Cran, M, E. Krafft, Angew. Chem. Int. Ed. 2012, 51, 9398. (c) Y. Yu, W. Yang, F. Rominger, A. S. K. Hashmi, Angew. Chem. Int. Ed. 2013, 52, 7586. (d) M. Chen, J. Liu, L. Wang, X. Zhou, Y. Liu, Chem. Commun., 2013, 49, 8650. (e) S. Zhu, L. Wu, X. Huang, J. Org. Chem. 2013, 78, 9120. (f) L.-J. Wang, H.-T. Zhu, A.-Q. Wang, Y.-F. Qiu, X.-Y. Liu, Y.-M. Liang, J. Org. Chem. 2014, 79, 204. (g) N. Sun, M. Chen, Y. Liu, J. Org. Chem. 2014, 79, 4055. (h) A. Ghosh, A. Basak, K. Chakrabarty, B. Ghosh, G. K. Das, J. Org. Chem. 2014, 79, 5652. (i) J.-M. Yang, X.-Y. Tang, M. Shi, Chem. Eur. J. 2015, 21, 4534.

14. Wang, D.; Zhang, Y.; Harris, A.; Gautam, L. N. S.; Chen, Y.; X. Shi, Adv. Synth. Catal. 2011, 353, 2584.

15. (a) Yu, M.; Zhang, G.; Zhang, L. Org. Lett. 2007, 9, 2147. (b) Ye L.; Zhang, L. Org. Lett. 2009, 11, 3646. (c) Yu, M.; Zhang, G.; Zhang, L. Tetrahedron 2009, 65, 1846. (d) Teresa de Haro, T.; Nevado, C. Chem. Commun., 2011, 47, 248. (e) D’Oyley, J. M.; Aliev, A. E.; Sheppard, T. D. Angew. Chem. Int. Ed. 2014, 53, 10747. 
Scheme 1. $\mathrm{PicAuCl}_{2}$ and $\mathrm{TriaAuCl}_{2}$.
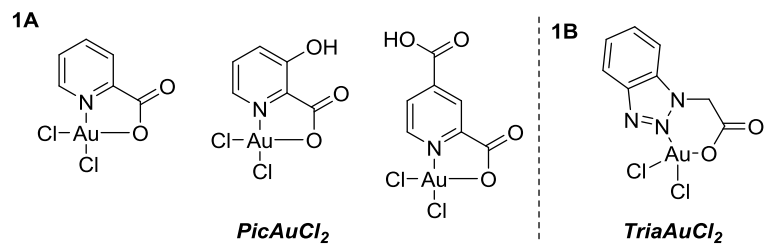

Scheme 2. The synthesis of TriaAu(III)Cl ${ }_{2}$ complex.

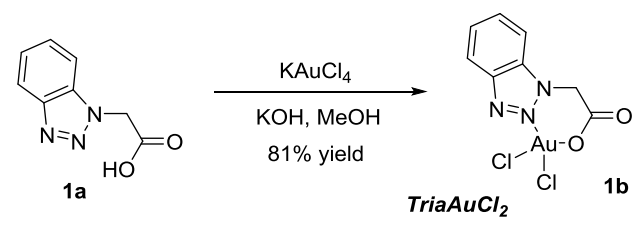

Scheme 3. Substrate experiment with alkyl-substituted propargyl alcohol.

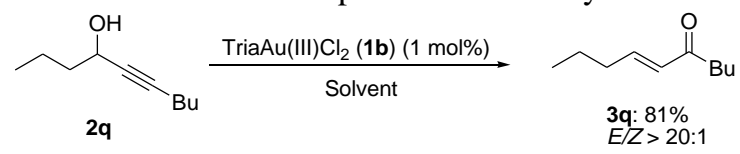


Table 1. Screening of reaction conditions ${ }^{a}$

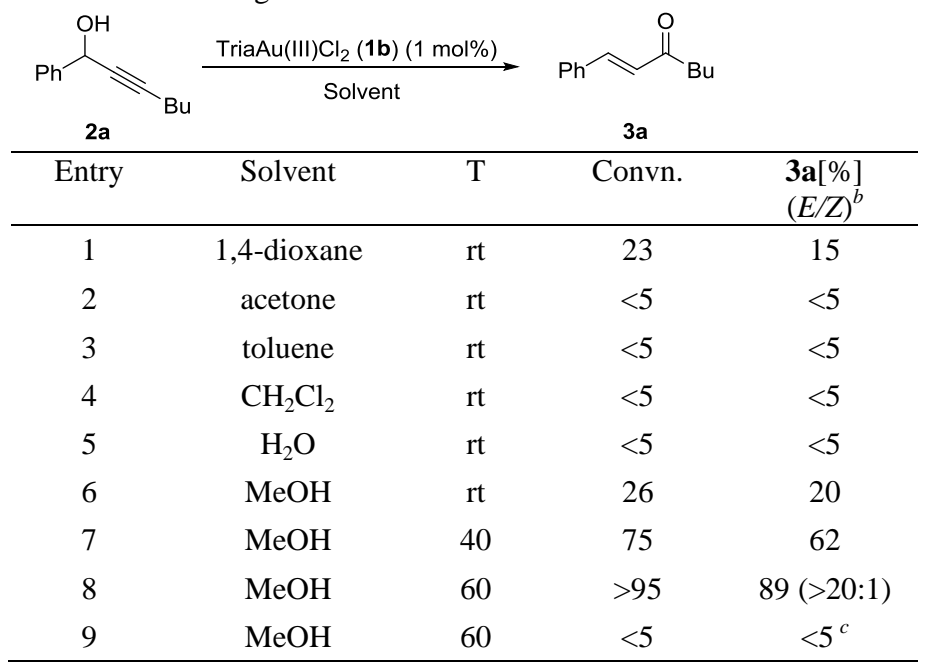

${ }^{a}$ Conditions: 2a $(0.5 \mathrm{mmol})$, TriaAu(III)Cl 2 (1b) $(1 \mathrm{~mol} \%)$, wet solvent $\left(10 \% \mathrm{H}_{2} \mathrm{O}\right), 6$ h. ${ }^{b}$ Isolated yields based on $2 \mathbf{a}$. ${ }^{c} \mathrm{No}[\mathrm{Au}]$ was used.

Table 2. Substrate expansion of propargyl alcohols ${ }^{a, b}$

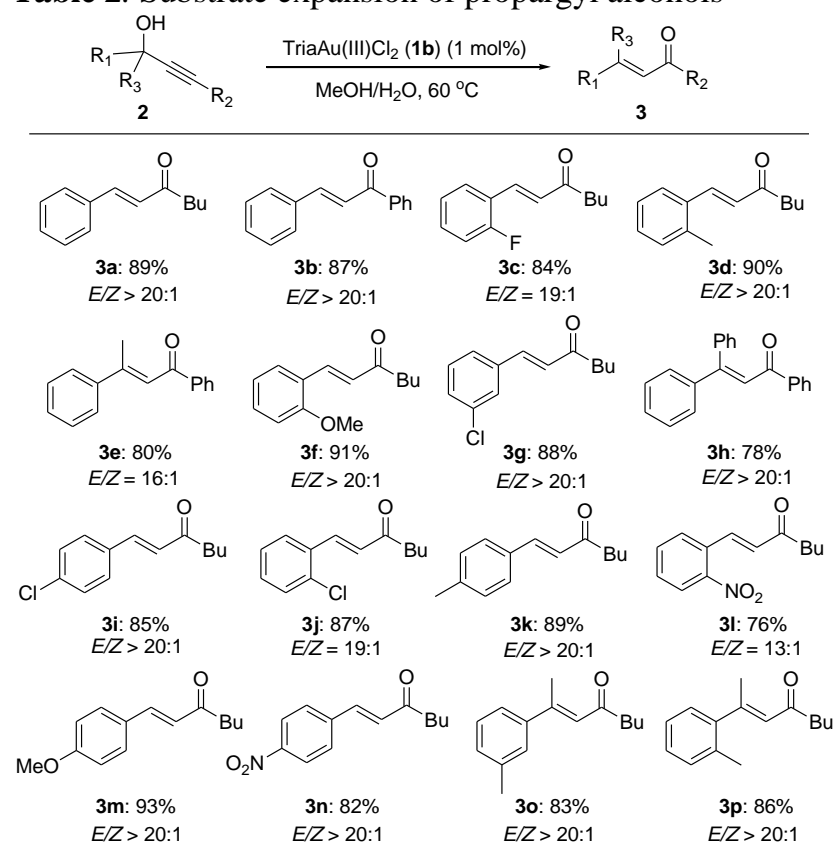

${ }^{a}$ Conditions: $2(0.5 \mathrm{mmol})$, TriaAu(III)Cl $\mathrm{Cl}_{2}(\mathbf{1 b})(1 \mathrm{~mol} \%), \mathrm{MeOH}\left(10 \% \mathrm{H}_{2} \mathrm{O}\right), 60{ }^{\circ} \mathrm{C}, 6 \mathrm{~h} .{ }^{b}$ Isolated yields based on 2.

Table 3. Substrate expansion of propargyl esters ${ }^{a, b}$ 


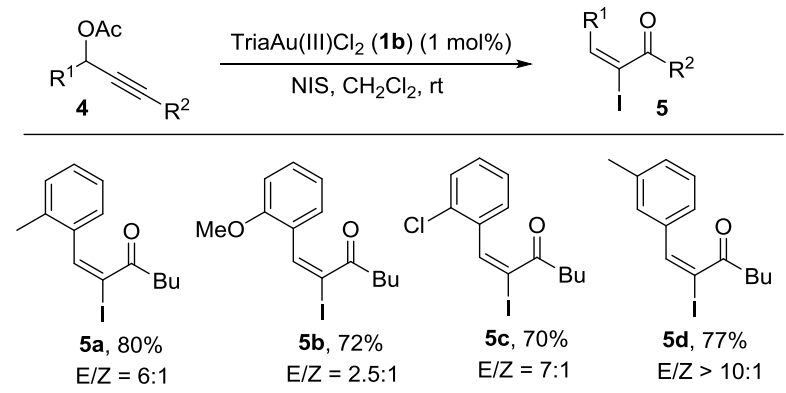

${ }^{a}$ Conditions: 4 (0.5 mmol), NIS (0.75 mmol), TriaAu(III) $\mathrm{Cl}_{2}(\mathbf{1 b})(1 \mathrm{~mol} \%), \mathrm{CH}_{2} \mathrm{Cl}_{2}(3 \mathrm{~mL}), 12 \mathrm{~h}$, rt. ${ }^{b}$ Isolated yields based on 4 . 


\section{Graphical Abstract}

To create your abstract, type over the instructions in the template box below.

Fonts or abstract dimensions should not be changed or altered.

\section{Triazole Acetyl Gold(III) Catalyzed Meyer-Schuster Rearrangement of Propargyl Alcohols}

Yongchun Yang, ${ }^{a}$ Yanan Shen, ${ }^{a}$ Xiaoli Wang, ${ }^{a}$ Yao Zhang, ${ }^{a}$ Dawei Wang*a and Xiaodong Shi*b

${ }^{a}$ The Key Laboratory of Food Colloids and Biotechnology, Ministry of Education, School of Chemical and Material Engineering, Jiangnan University, Wuxi 214122, Jiangsu Province, China. ${ }^{b} 4202$ E Fowler Ave, Department of chemistry, University of South Florida, Tampa, Florida, USA.

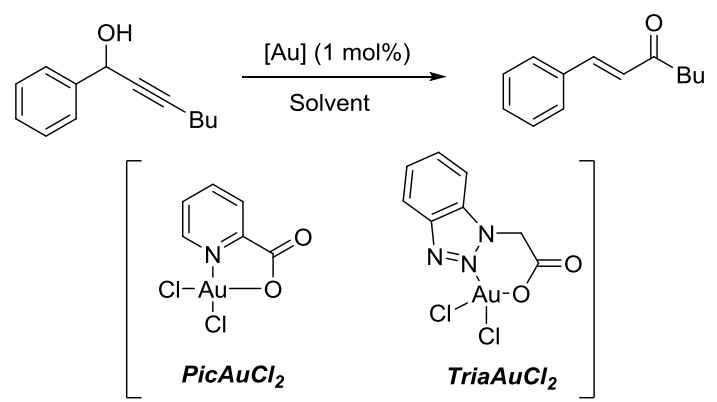

A new type triazole acetyl gold(III) was prepared and found to be an effective catalyst in Meyer-Schuster rearrangement of propargyl alcohols. The reactions proceeded well under much milder conditions to afford enones bearing a wide range of functional groups, thereby opening a new avenue for gold(III) catalysis. In addition, $\mathrm{TriaAuCl}_{2}$ catalyst was also effective on promotion of a-haloenones synthesis. 